\title{
Neoadjuvant endocrine therapy: for whom, for how long?
}

\author{
Fatima Cardoso
}

$\mathrm{N}$ eoadjuvant, primary or preoperative systemic therapy (PST) has long been the standard of care for locally advanced or inoperable breast cancer. Landmark trials, such as the NSABP-B18 [1], and meta-analysis have proven that PST for operable early breast cancer leads to the same long-term outcomes (disease-free and overall survival) with only a minor increase in local recurrence. Over the decades PST has been used not only for downstaging and increase of breast-conserving surgery but also as an in vivo assessment of response to therapy with the aim of helping to define the best adjuvant systemic therapy. Furthermore, many studies have shown that the achievement of a pathological complete response (pCR) after neoadjuvant chemotherapy is an excellent prognostic factor. More recently, several studies, such as the M.D. Anderson series, the I-SPY 1 study and the German meta-analysis of neoadjuvant chemotherapy, have shown that responses to PST are intrinsically dependent on the molecular subtype of breast cancer, with triple-negative and HER-2-positive subtypes obtaining the highest responses. On the other hand, the luminal A subtype rarely achieves a pCR with neoadjuvant chemotherapy but in this case there is no negative impact on long-term outcome since this subtype of breast cancer derives the highest benefit from endocrine therapy.

Previous studies have evaluated the role of neoadjuvant or preoperative endocrine therapy (PET) in operable hormone receptor (HR)-positive early breast cancer, some comparing the efficacy of tamoxifen and aromatase inhibitors (e.g., the P024 letrozole trial [2], the IMPACT [3] and PROACT [4] trials). In most of these studies the duration of neoadjuvant therapy was 3-4 months, decided arbitrarily in part due to the fear of progressive disease and loss of operability. The article by Llombart Cussac and colleagues addresses the important issue of duration of PET.

F. Cardoso (四)

Breast Cancer Unit

Champalimaud Cancer Center

Lisbon, Portugal

e-mail: fatimacardoso@fundacaochampalimaud.pt

Despite being a small (70 patients) non-randomised phase II study, some interesting findings are worth noticing. The mean time to first response was 3.9 months and the median time to maximum response 4.2 months. While a third of responders achieved maximum response beyond 6 months of treatment, very few responders were identified after 6 months and none from 8 to 12 months [5]. Together with data from other PET studies, these data reinforce the notion that endocrine therapy takes longer to achieve responses than chemotherapy but that most responders can be identified within the first 4 months of treatment; data also suggest that some patients require longer duration of treatment to achieve the highest benefit, although for the majority a duration of 6 months seems enough. In a previous study by Dixon and colleagues the issue of optimal duration of PET was also addressed, with similar results: continuing letrozole in responding patients beyond 3-4 months achieves further clinical reduction in tumour size, increasing the number of breast-conserving surgeries [6]. All these studies emphasise the safety of PET beyond the traditionally used 3-4 months.

In the last decade, there has been a change in treatment decision making for early breast cancer, with biology taking centre stage and staging (tumour size and nodal status) second place. Even if one does not have access to the more sophisticated but also more expensive genomic tools (i.e., MammaPrint ${ }^{\mathrm{TM}}$, Oncotype $\mathrm{DX}^{\mathrm{TM}}$ or Genomic Grading), one can use several biomarkers to estimate the potential benefit of adjuvant chemotherapy [7]. The most commonly used biomarkers are ER, PgR, HER-2 and Ki67, and a combination of these can be used as immunohistochemistry surrogates of molecular subtypes. Additionally, histology is important since lobular cancers almost never achieve a pCR with neoadjuvant chemotherapy [8] but they have very good outcomes with optimal endocrine therapy. There is however a substantial number of patients with HR-positive tumours with intermediate risk characteristics for whom the estimation of the added benefit of chemotherapy is very difficult to ascertain. In these cases, the use of PET is of extreme value since if a very good response is obtained, the added benefit of adjuvant chemotherapy is probably very low and over-treatment may be avoided. The possibility of 
verifying the in vivo response to endocrine therapy gains even higher importance due to the lack of validated predictive markers of response/resistance to endocrine agents, apart from the presence and the level of HR. Previous studies have suggested that early (about 2 weeks) changes in Ki67 status may be an indicator of response to PET [9] but need to be confirmed in larger studies. Preliminary data from the 3 PET studies mentioned above suggested that HER-2 status could be a differentiator between response to tamoxifen and to aromatase inhibitors. These findings did not hold true in larger adjuvant studies and at the present time there is no reliable biomarker to differentiate response to tamoxifen and aromatase inhibitors, making this a research priority. The major weakness of the study by Llombart Cussac et al. [5] is the lack of biomarker evaluation. Only ER and PgR status are reported, with a notion of higher response with higher levels of receptors. We cannot however tease out luminal A from luminal B cancers in this study, although due to the age eligibility criteria (over 65) one might expect that the majority of these breast cancers were luminal A. Biomarkers would have also been important to characterise patients who respond earlier and faster (for whom 4 months of PET are sufficient) from those who need longer duration of this treatment.
One of the major challenges with PST is how to best monitor responses. Clinical measurements lack accuracy and serial mammographic assessment exposes patients to substantial doses of radiation. Serial ultrasound measurements are frequently used but are also radiologistdependent. Breast MRI, particularly functional MRI, has gained increasing acceptance, particularly in cases where traditional breast imaging is less performing, such as with lobular cancers and younger women, but it is also associated with increasing costs.

Finally, a very important point to consider is the impact of PST on the biology of the breast tumour. In the study by Ellis et al. [10], some luminal A tumours were changed to luminal B after treatment with neoadjuvant letrozole. Independently of the mechanism of this change (true change in biology, clone selection or other) this observation underlines the importance of reassessing tumour biology after PST. During this period of economic constraints, in many centres physicians must choose to have biomarkers assessed in either the biopsy or the surgical specimen. However, for a correct decision of the optimal adjuvant systemic therapy, it may be crucial to take into account the initial biomarker status and the post-neoadjuvant treatment status.

\section{References}

1. Fisher B, Brown A, Mamounas E et al (1997) Effect of preoperative chemotherapy on localregional disease in women with operable breast cancer: findings from the National Surgical Adjuvant Breast and Bowel Project B-18. J Clin Oncol 15:2483-2493

2. Ellis M, Ma C (2007) Letrozole in the neoadjuvant setting: the P024 trial. Breast Cancer Res Treat 105:33-43

3. Smith IE, Dowsett M, Ebbs SR et al; IMPACT Trialists Group (2005) Neoadjuvant treatment of postmenopausal breast cancer with anastrozole, tamoxifen, or both in combination: the Immediate Preoperative Anastrozole, Tamoxifen, or Combined with Tamoxifen (IMPACT) multicenter double-blind randomized trial. J Clin Oncol 23: 5108-5116

4. Cataliotti L, Buzdar AU, Noguchi S et al (2006) Comparison of anastrozole versus tamoxifen as preoperative therapy in postmenopausal women with hormone receptor-positive breast cancer: the Pre-Operative "Arimidex" Compared to Tamoxifen (PROACT) trial. Cancer 106:20952103

5. Llombart-Cussac A, Guerrero A, Galán A et al (2012) Phase II trial with letrozole to maximum response as primary systemic therapy in postmenopausal patients with ER/PgR $[+]$ operable breast cancer. Clin Transl Oncol 14:125-131

6. Dixon JM, Renshaw L, Macaskill EJ et al (2009) Increase in response rate by prolonged treatment with neoadjuvant letrozole. Breast Cancer Res Treat 113:145-151
7. Bedard PL, Cardoso F (2011) Can some patients avoid adjuvant chemotherapy for early-stage breast cancer? Nat Rev Clin Oncol 8:272-279

8. Colleoni M, Bagnardi V, Rotmensz N et al (2009) Increasing steroid hormone receptors expression defines breast cancer subtypes non responsive to preoperative chemotherapy. Breast Cancer Res Treat 116:359-369

9. Dowsett M, Smith IE, Ebbs SR et al; IMPACT Trialists Group (2007) Prognostic value of Ki67 expression after short-term presurgical endocrine therapy for primary breast cancer. J Natl Cancer Inst 99:167-170

10. Ellis MJ, Tao Y, Luo J et al (2008) Outcome prediction for estrogen receptor-positive breast cancer based on postneoadjuvant endocrine therapy tumor characteristics. J Natl Cancer Inst 100:1380-1388 